Jânio Monteiro • António João Silva · António Mortal · Jaime Aníbal . Manuela Moreira da Silva - Miguel Oliveira . Nelson Sousa Editors
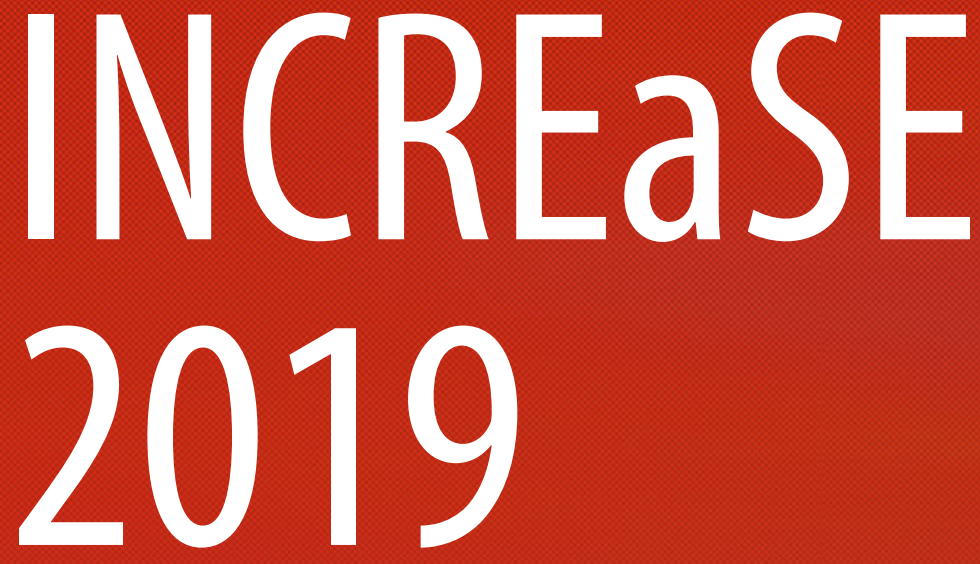

Proceedings of the 2 nd International Congress on Engineering and Sustainability in the XXI Century 
Jânio Monteiro · António João Silva ·

António Mortal · Jaime Aníbal .

Manuela Moreira da Silva .

Miguel Oliveira $\cdot$ Nelson Sousa

Editors

\section{INCREaSE 2019}

Proceedings of the 2nd International Congress

on Engineering and Sustainability

in the XXI Century 
Editors

Jânio Monteiro

Instituto Superior de Engenharia

Universidade do Algarve

Faro, Portugal

António Mortal

Instituto Superior de Engenharia

Universidade do Algarve

Faro, Portugal

Manuela Moreira da Silva

Instituto Superior de Engenharia

Universidade do Algarve

Faro, Portugal

Nelson Sousa

Instituto Superior de Engenharia

Universidade do Algarve

Faro, Portugal
António João Silva

Instituto Superior de Engenharia

Universidade do Algarve

Faro, Portugal

Jaime Aníbal

Instituto Superior de Engenharia

Universidade do Algarve

Faro, Portugal

Miguel Oliveira

Instituto Superior de Engenharia

Universidade do Algarve

Faro, Portugal

ISBN 978-3-030-30937-4

ISBN 978-3-030-30938-1 (eBook)

https://doi.org/10.1007/978-3-030-30938-1

(C) Springer Nature Switzerland AG 2020

This work is subject to copyright. All rights are reserved by the Publisher, whether the whole or part of the material is concerned, specifically the rights of translation, reprinting, reuse of illustrations, recitation, broadcasting, reproduction on microfilms or in any other physical way, and transmission or information storage and retrieval, electronic adaptation, computer software, or by similar or dissimilar methodology now known or hereafter developed.

The use of general descriptive names, registered names, trademarks, service marks, etc. in this publication does not imply, even in the absence of a specific statement, that such names are exempt from the relevant protective laws and regulations and therefore free for general use.

The publisher, the authors and the editors are safe to assume that the advice and information in this book are believed to be true and accurate at the date of publication. Neither the publisher nor the authors or the editors give a warranty, expressed or implied, with respect to the material contained herein or for any errors or omissions that may have been made. The publisher remains neutral with regard to jurisdictional claims in published maps and institutional affiliations.

This Springer imprint is published by the registered company Springer Nature Switzerland AG

The registered company address is: Gewerbestrasse 11, 6330 Cham, Switzerland 


\title{
Organization
}

INCREaSE is an international congress organized by the Engineering Institute from the University of Algarve, Faro, Portugal.

\section{Coordinating Committee}

\author{
António Mortal (Director) \\ Manuela Moreira da Silva \\ (President of the \\ Technical-Scientific Council) \\ João Ataíde \\ (Portuguese Secretary of State \\ for the Environment) \\ Paulo Águas (Rector) \\ Adelino Canário \\ Anabela Romano \\ António Ruano \\ António Tadeu \\ Armando Silva Afonso \\ Augusto Ferreira Guedes \\ Carlos Alberto Aires \\ Francisco Serra \\ João Guerreiro \\ Joaquim Peres \\ Luciano Lourenço \\ Luís Chícharo \\ Maria João Bebianno \\ Patrícia Pinto \\ Rogério Bacalhau \\ Universidade do Algarve, Instituto Superior \\ de Engenharia, Portugal \\ Universidade do Algarve, Instituto Superior \\ de Engenharia, Portugal \\ Ministry of the Environment and Energy \\ Transition, Portugal \\ Universidade do Algarve, Portugal \\ CCMAR, Portugal \\ MeditBio, Portugal \\ CINTAL, Portugal \\ ITeCons, Portugal \\ ANQIP, Portugal \\ Ordem dos Engenheiros Técnicos, Portugal \\ Ordem dos Engenheiros, Portugal \\ CCDR Algarve, Portugal \\ CRIA, Portugal \\ Águas do Algarve, S.A., Portugal \\ RISCOS, Portugal \\ UNESCO Chair in Ecohydrology, Portugal \\ CIMA, Portugal \\ CIEO, Portugal \\ C. M. Faro, Portugal
}


Susana Neto

Vítor Aleixo

\section{Organizing Committee}

António João Silva

Elisa Silva

Isabel Ratão

Jaime Aníbal

Jânio Monteiro

Miguel Oliveira

Nelson Sousa

Paulo Santos

André Botelheiro (Director of Communication Office)

Ana Paula Ferreira (Director of Technical Services)

\section{Invited Speakers}

Andrew Patton McCoy

Edmilson Teixeira

Filipe Duarte do Santos

Tiago Rebelo

Isabel Ferreira

Stephen Thomas

\section{Scientific Committee}

Aicha Nancib

Aires Camões
APRH, Portugal

C. M. Loulé, Portugal
Universidade do Algarve, Instituto Superior de Engenharia, Portugal

Universidade do Algarve, Instituto Superior de Engenharia, Portugal

Universidade do Algarve, Instituto Superior de Engenharia, Portugal

Universidade do Algarve, Instituto Superior de Engenharia, Portugal

Universidade do Algarve, Instituto Superior de Engenharia, Portugal

Universidade do Algarve, Instituto Superior de Engenharia, Portugal

Universidade do Algarve, Instituto Superior de Engenharia, Portugal

Universidade do Algarve, Instituto Superior de Engenharia, Portugal

Universidade do Algarve, Portugal

Universidade do Algarve, Portugal

Virginia Polytechnic Institute and State University, USA

Federal University of Espírito Santo State, Brazil

Faculty of Sciences, University of Lisbon, Portugal

CEIIA, Portugal

Instituto Politécnico de Bragança, Portugal OGI, Groundwater Specialists Ltd.,

Durham University, UK

Ferhat Abbas University, Setif 1, Algeria

University of Minho, Portugal 
Ana Cristina Figueira

Ana Teresa Lima

Anabela Rebelo

Andrew McCoy

Antonio Illana

António Martins

António Matias

António Ruano

Arturo Aquino Martín

Aureliano Alves

Aurizia Anica

Carla Antunes

Carla Rodrigues

Carlos Otero da Silva

Celestina Pedras

Celestino Ruivo

Célia Quintas

Célia Ramos

Clauciana Schmidt Bueno de Moraes

Cláudia de Almeida

Cláudia Sequeira

Cristiano Cabrita

Damian Beben

Duarte Nuno Duarte

Edmilson Teixeira

Eduardo Esteves
Universidade do Algarve, ISE and CIEO, Portugal

Universidade Federal do Espírito Santo, Brasil and University of Waterloo, ON, Canada

Agência Portuguesa do Ambiente, Portugal

Virginia Polytechnic Institute and State University, USA

Universidad de Cádiz, Spain

Grupo Águas de Portugal, Águas do Algarve, Portugal

Universidade do Algarve, FE and CIEO, Portugal

Universidade do Algarve, FCT and CINTAL, Portugal

University of Huelva, Spain

Universidade do Algarve, FCT and CCMAR, Portugal

Universidade do Algarve, ESEC and IELT, Portugal

Universidade do Algarve, FCT and MeditBio, Portugal

Universidade de Aveiro e ANQIP, Portugal

Universidade do Algarve, ISE, Portugal

Universidade do Algarve, FCT and LEAF, Portugal

Universidade do Algarve, ISE, Portugal

Universidade do Algarve, ISE and MeditBio, Portugal

Universidade do Algarve, ESGHT, Portugal

Universidade Estadual Paulista, Brazil

Universidade do Algarve, ESGHT and CIEO, Portugal

Universidade do Algarve, ISE and CIMA, Portugal

Universidade do Algarve, ISE, Portugal

Opole University of Technology, Poland

Universidade do Algarve, FCT and CIMA, Portugal

Universidade Federal do Espírito Santo, Brazil

Universidade do Algarve, ISE and CCMAR, Portugal 
Eduardo López González

Eladio Durán Aranda

Enzo Martinelli

Eugénia Ferreira

Eusébio Conceição

Fabian Sá

Fátima Farinha

Fernando Branco

Fernando Cánovas

Fernando Martins

Flávio Martins

Francisca Segura

Francisco Guzmán Navarro

Francisco Javier Vicario Llerena

Gabriela Schutz

Gil Fraqueza

Giovanna Concu

Gonçalo Prates

Gorete Dinis

Helena Fernandez

Hugo Pinto

Isménio Martins

Jacobo Porteiro

Jeff Camkin

Jessie Melo

João Castro Gomes

João Estevão
Instituto Nacional de Técnica Aeroespacial, Spain

University of Huelva, Spain

University of Salerno, Italy

Universidade do Algarve, Faculdade de Economia, Portugal

Universidade do Algarve, FCT and CINTAL, Portugal

Universidade Federal do Espírito Santo, Brazil

Universidade do Algarve, ISE and CEPAC, Portugal

University of Coimbra, Portugal

Universidad Católica San Antonio de Murcia, Spain

Universidade do Algarve, ISE and CIEO, Portugal

Universidade do Algarve, ISE and CIMA, Portugal

University of Huelva, Spain

University of Malaga, Spain

Universidad de Cádiz, Spain

Universidade do Algarve, ISE and CEOT, Portugal

Universidade do Algarve, ISE and CCMAR, Portugal

University of Cagliari, Italy

Universidade do Algarve, ISE and CEG, Portugal

Instituto Politécnico de Portalegre and GOVCOPP, Portugal

Universidade do Algarve, ISE and CIEO, Portugal

Universidade de Coimbra and CES; Universidade do Algarve, FE, Portugal

Universidade do Algarve, ISE, Portugal

University of Vigo, Spain

CENRM and University of Western Australia, Australia

Universidade do Algarve, ISE and MeditBio, Portugal

University of Beira Interior, Portugal

Universidade do Algarve, ISE and CIMA Portugal 
João M. F. Rodrigues

Jorge de Brito

Jorge Isidoro

Jorge Pereira

José António Monteiro

José Carlos Teixeira

José Luís Argain

José Paulo Monteiro

Juan Jose Salas

Juan Manuel Enrique Gomez

Katherine Flynn

Kinga Krauze

Lahcen Boukhattem

Ludovina Galego

Luís Fialho

Luís Oliveira

Manuel Duarte Pinheiro

Manuela Rosa

Marcos Mateus

Margarida Ribau Teixeira

Margarida Vieira

Maria de Belém Costa

María Martín Morales

Mariana Golumbeanu

Mário Costa

Mário Serafim Nunes
Universidade do Algarve, ISE and LARSyS, Portugal

Instituto Superior Técnico, University of Lisbon, Portugal

Universidade do Algarve, ISE and MARE, Portugal

Universidade do Algarve, ISE and MeditBio, Portugal

Universidade do Algarve, FCT and CEPAC, Portugal

Universidade do Minho, Portugal

Universidade do Algarve, FCT and CIMA, Portugal

Universidade do Algarve, FCT and CERIS, Portugal

Centre for New Water Technologies (CENTA), Spain

University of Huelva, Spain

The European Association for Food SafetySAFE consortium, France

European Regional Centre for Ecohydrology of the Polish Academy of Sciences, Poland

University of Cadi Ayyad, Morocco

Universidade do Algarve, ISE and MeditBio, Portugal

University of Evora, Portugal

Universidade do Algarve, ISE, Portugal

Instituto Superior Técnico, University of Lisbon, Portugal

Universidade do Algarve, ISE and CIEO, Portugal

IST, University of Lisbon and MARETEC, Portugal

Universidade do Algarve, FCT and CENSE, Portugal

Universidade do Algarve, ISE and MeditBio, Portugal

Universidade do Algarve, FCT and MeditBio, Portugal

University of Granada, Spain

Balkan Environmental Association (BEnA), Romania

Instituto Superior Técnico, IDMEC, University of Lisbon, Portugal

INESC Inovação, Lisbon, Portugal 
Marisol Correia

Mercedes Gonzalez Wanguemert

Miguel Angel Ridao

Miguel Reimão Costa

Natividade Vieira

Olfa Ben Said

Patrícia Nunes

Paulo Felisberto

Paulo Relvas

Pedro J. S. Cardoso

Rafaela Matos

Raúl Barros

Rijkelt Beumer

Roberto Lam

Rodrigo Braga Moruzzi

Rui Cruz

Rui Lança

Rute Rocha

Sara Raposo

Senhorinha Teixeira

Sérgio de Jesus

Stephen Thomas

Tomás Figueiredo

Vítor Gonçalves
Universidade do Algarve, ESGHT, Portugal

Guatizamar S.L e CCMAR, Portugal

University of Seville, Spain

Universidade do Algarve, FCT and CEPAC, Portugal

University of Porto, Portugal

Bizerta Faculty of Sciences, Carthage University, Tunisia

Universidade do Algarve, ISE and MeditBio, Portugal

Universidade do Algarve, ISE and LarSyS, Portugal

Universidade do Algarve, FCT and CIMA, Portugal

Universidade do Algarve, ISE, and LARSyS, Portugal

Laboratório Nacional de Engenharia Civil, Portugal

Universidade do Algarve, FCT and CIMA, Portugal

Department of Agrotechnology and Food Sciences, Wageningen University and Research, The Netherlands

Universidade do Algarve, ISE and LARSyS, Portugal

Universidade Estadual Paulista, Brazil

Universidade do Algarve, ISE and MeditBio, Portugal

Universidade do Algarve, ISE, Portugal

Universidade do Algarve, ESEC and DESYM, Portugal

Universidade do Algarve, FCT and CIMA, Portugal

Universidade do Minho, Portugal

Universidade do Algarve, FCT and LARSyS, Portugal

OGI Groundwater Specialists Ltd., Durham University, UK

Instituto Politécnico de Bragança and CIMO, Portugal

Universidade dos Açores and CIBIO, Azores, Portugal 


\section{Institutional Support}

Universidade do Algarve

UNESCO, UniTwin, Chair on Ecohydrology: Water for Ecosystems and Societies CIMA-Universidade do Algarve

ITeCons-Instituto de Investigação e Desenvolvimento Tecnológico para a Construção, Energia, Ambiente e Sustentabilidade

Associação Portuguesa de Recursos Hídricos

RISCOS-Associação Portuguesa de Riscos, Prevenção e Segurança

Ordem dos Engenheiros

\section{Sponsors}

Fundação para a Ciência e a Tecnologia (FCT)

Ordem dos Engenheiros da Região Sul

OGI-Groundwater Specialists

Boode-Waterwell Systems

PLATIPUS-Earth Anchoring Systems

Águas do Algarve

Câmara Municipal de Faro

Câmara Municipal de Loulé

CACIAL-Cooperativa Agrícola de Citricultores do Algarve

\section{Media Partner}

Sul Informação 


\title{
Two-Phase Bubble Flow: Experimental and Numerical Challenges
}

\author{
Gláucio Kenji Matoba ${ }^{1(\bowtie)}$, Luis Frölén Ribeiro ${ }^{2,3}$, Valdemar Garcia $^{4}$, \\ and Fábio Kenji Suguimoto ${ }^{5}$ \\ 1 Bragança Polytechnic Institute/Federal University of Technology - Paraná, \\ Cornélio Procópio, Brazil \\ matoba@alunos.utfpr.edu.br \\ 2 Mechanical Technical Department, Bragança Polytechnic Institute, \\ Bragança, Portugal \\ frolen@ipb.pt \\ 3 Centre for Renewable Energy Research, INEGI, \\ Porto, Portugal \\ 4 Mechanical Applied Department, Bragança Polytechnic Institute, \\ Santa Apolónia Campus, Bragança, Portugal \\ valdemar@ipb.pt \\ 5 Mechanical Engineering Department, Federal University of Technology - Paraná, \\ Cornélio Procópio, Brazil \\ fksuguimoto@utfpr.edu.br
}

\begin{abstract}
A water and air mixture model is validated via experimental data in a newly rebuild laboratory apparatus where the main variable compared was the pressure gradient along the pipe in a bubble flow pattern. The experimental apparatus presents a constant circular crosssection of $0.032 \mathrm{~m}$ of diameter and $3.815 \mathrm{~m}$ of length. The flow set-up was ascendant co-current and 30 pairs of superficial velocities of liquidgas were measured. A numerical solution, using the mixture model, was implemented to predict the pressure gradient and void fraction of the two-phase flow for a one dimensional, steady-state, isothermal, no phase transition, no mass transfer and constant specific mass and viscosity conditions. The pressure gradient numerical results of the mixture model agreed with experimental data within an relative error envelope inferior to $2 \%$.
\end{abstract}

Keywords: Two-phase flow $\cdot$ Mixture model $\cdot$ Pressure gradient · Two-phase flow experimental apparatus

\section{Introduction}

Two-phase flows of gas-liquid mixtures in a vertical pipes has an extensive application in the chemical, petroleum and energy production industries.

In nuclear reactors, for instance, an important parameter is the drop pressure 
which can, significantly, affect the safety of nuclear reactors. The accident Loss of Coolant Accidents (LOCA) are mainly due to small crevasses created in the reactor's wall [1].

Another application for two-phase flow study is the air-lift pump. The air-lift pumps have several advantages over the other pumps, to mention a few they do not have any moving parts and do not need lubrication or suffering from wearing out problem, which means low maintenance are required. Air-lift pumps can be used for lifting corrosive and/or toxic substances in chemical industries, sandy or salty water and viscous liquids like hydro-carbons in oil industry [2]. Air-lift pumps are finding increasing use when a compressed air is readily available as a source of renewable energy for water pumping applications [3].

Other important and fundamental parameter for two-phase flow modeling is the void fraction. The void fraction is essential for calculation of many parameters, such as two-phase mixture densities, viscosities, actual velocities of each phase and the frictional pressure-drop. There are some experimental methods to obtain the void fraction: electrical impedance tomography $[4,5]$; double wire mesh sensors [6] and quick-closing ball valve technique [7].

There are 4 main types of two-phase flow classified according to the increase in the gas superficial velocity [8]: bubble, slug, churn and annular flow. The experimental apparatus that will be presented allows the study of these four types of flows. However, in this paper we present the study of an upward flow of air and water in steady-state, one-dimensional on a vertical pipe with constant diameter. This work addresses 2 vectors of the bubble flow regime and pattern: the experimental challenges related to the study of two-phase flows; the verification of the simplified numerical model $[9,10]$ against the experimental results.

The bubble flow regime occurs when the gas phase is approximately uniformly distributed in the form of discrete bubble in a continuous liquid phase [8]. The relevant parameters for the evaluation of this type of flow are the pressure gradient $\Delta P / L$ and the void fraction $\alpha$, defined as the drop pressure between two distanced points and the gas fraction in the pipe. These variables will be addressed in Sects. 2.1 and 2.2.

The experimental procedures and problems to obtain bubble flow accurate measurements are similar to the other types of flows. These challenges are also discussed in this paper in Sect.4.3.

\section{Experimental Procedure}

The experiments were performed in a vertical acrylic pipe with a constant circular cross-section of $0.032 \mathrm{~m}$ and $3.815 \mathrm{~m}$ of length, named bubble column. The pressure difference was measured with a differential manometer, DM, connected to 2 pressure taps, $\mathrm{P} 1$ and $\mathrm{P} 2$, located $3.560 \mathrm{~m}$ apart: at the base and the top of the bubble column. The experimental apparatus is shown schematically in Fig. 1.

The bubble column have also two ball valves distanced $1.165 \mathrm{~m}$ apart to measure the average void fraction, V1 and V2 in Fig. 1. The blue line in Fig. 1 


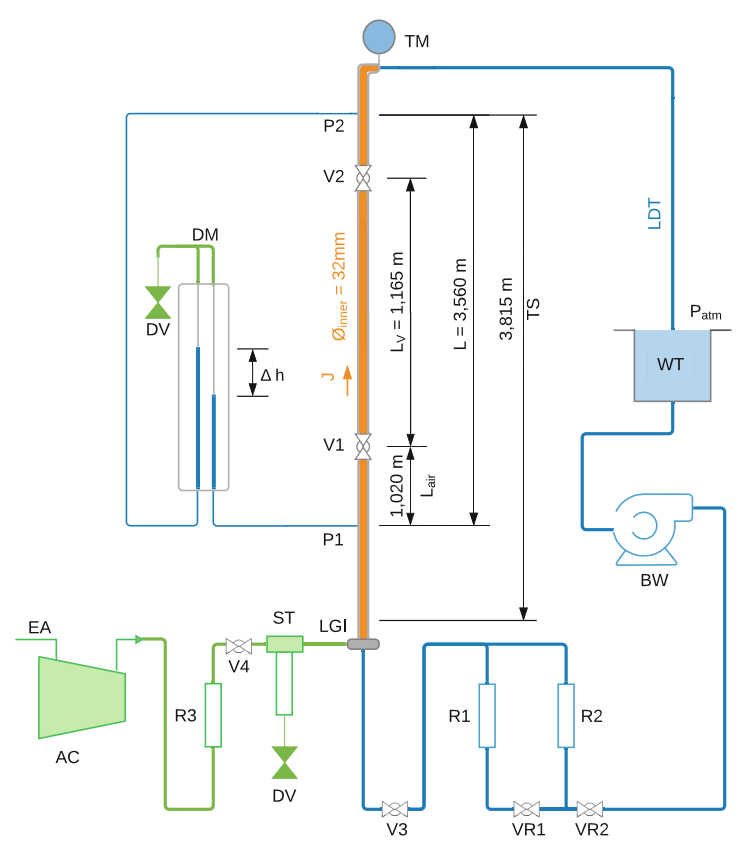

(a)

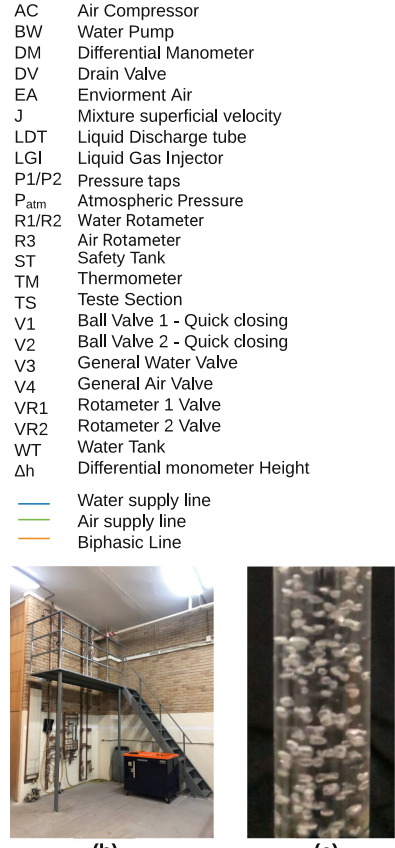

(b)

(c)

Fig. 1. (a) Schematic representation of the experimental apparatus, (b) Experimental apparatus, (c) Experimental bubble flow.

represents the water circuit. Water is introduced at the base of the column via a centrifugal pump BW. The flow rate is regulated by a ball valve VR1 or VR2, and measured by a rotameter R1 (KDG - Houdec S7E-60) or R2 (RDG - Houdec S18xE500), with a $3.00 \%$ accuracy. The air, represented by green line in Fig. 1, is supplied by a air compressor AC, with maximum pressure of $6.00 \times 10^{5} \mathrm{~Pa}$. The air flow rate is controlled by a rotameter R3 (KDG-1100 - 2C-150 - ruby ball), which allows a max and min flow rate, respectively, of $1.00 \times 10^{-5}$ and $1.67 \times 10^{-5} \mathrm{~m}^{3} / \mathrm{s}$ with an accuracy of $5.00 \%$. The gas was introduced in the base of the column through a sparging device, LGI. This device was made with porous nylon material, allowing the formation of small-diameter $(\sim 0.003 \mathrm{~m})$ bubbles and proper mixing with the water. In order to prevent water from entering the air circuit by accident and hampered the measurement of the air flow, a safety tank, ST, was built.

Thirty pairs of superficial velocities were tested, which were 6 superficial liquid velocities, $J_{L}$, between $1.720 \times 10^{-2}$ and $10.360 \times 10^{-2} \mathrm{~m} / \mathrm{s}$ with 5 superficial gas velocities, $J_{G}$, between $1.243 \times 10^{-2}$ and $2.072 \times 10^{-2} \mathrm{~m} / \mathrm{s}$. For each pair of superficial velocities, the pressure gradient, $\Delta P / L$, and the void fraction, $\alpha$, were measured. The temperature at which the experiments were performed varied between 17.5 and $18.5^{\circ} \mathrm{C}$, measured in the end of the test section, TS, by a thermometer, TM. 


\subsection{Experimental Drop Pressure}

For each pair of superficial velocities $J_{L}$ and $J_{G}$, and after steady state was reached, the value of the water height difference was recorded in the differential manometer, $\Delta h=h_{2}-h_{1}$. The difference of pressure, $\Delta P$, or pressure gradient, $\Delta P / L$, its derived from the hydrostatic equation, Eq. (1).

$$
\frac{P 1-P 2}{L}=\frac{\Delta P}{L}=\rho_{L} g-\frac{\rho_{L} g \Delta h}{L}
$$

where $L$ is the distance between the two taps in the column, $\rho_{L}$ is the water density and $g$ is the acceleration of gravity.

\subsection{Experimental Void Fraction}

The void fraction was obtained by two methods: the first through the quickly closing of the valves $V 1$ and $V 2$; the second using the relation from [11], recurring to the experimental terminal rise bubble velocity in a stagnant medium.

The first method is simple and functional [12], the ball valves were used to completely stop the two-phase flow. Once the two valves are closed simultaneously, it is possible to measure the gas height $\left(L_{\text {gas }}\right)$, and then calculate the void fraction as the ratio of heights between the gas and the total height $\left(L_{V}\right)$, Eq. (2).

$$
\alpha_{\text {exp }}=\frac{L_{g a s}}{L_{V}}
$$

This method is known as the quick-closing valves method.

The second method used the relation of Zuber [11], Eq. (3), modified by the works form Nicklin [13]. This is known as the Zuber/Nicklin relation, and is obtained from the rearrangement of the kinematic slip law:

$$
\alpha_{e x p}=\frac{J_{G}}{J+V_{\infty, B}}
$$

where $V_{\infty, B}$ is the terminal bubble velocity rise in a stagnant medium, experimentally measured through the length $L_{a i r}$ (shown in Fig. 1) and with the aid of a chronometer. $J=J_{G}+J_{L}$ is the superficial mixture velocity.

\section{$3 \quad$ Numerical Procedure}

\subsection{Mixture Model}

The mixture model is based on the simplification of the two-fluid model, which results from the application of the concept of mixing in a two-phase flow, i.e., the components of the mixture are treated as a single fluid with specific rheological characteristics [9]. An important aspect of the mixture model is the reduction in the number of transport equations required for the formulation as compared to the two-fluid model [9]. 
There are two variables to be determined in the mixture model: one relative to the void fraction and the terms that involve the surface tensions of the mixture. Therefore, it is necessary to introduce models for the determination of these closing parameters, which in turn depend on the flow pattern, in the case of bubble flow.

An alternative form of the mixture model, introduced by [10], proposes mass and momentum conservation equations for each phase of the mixture in terms of the primitive variables of the problem, rather than using the properties of the mixture and the diffusion velocity. This alternative form was developed for studies on two-phase flows in the steady-state and transient regime [10]. In this model, it is necessary to know the slip ratio between the phases for the closing of the system equations.

The main equations of the mixture model are based on the modified drift flux model [10]. The mass conservation equations of the gas and liquid phases are defined in Eqs. (4) and (5), respectively [10].

$$
\begin{gathered}
\frac{\partial}{\partial t}\left[\rho_{G} \alpha_{G}\right]+\frac{\partial}{\partial z}\left[\rho_{G} \alpha_{G} U_{G}\right]=\Gamma, \\
\frac{\partial}{\partial t}\left[\rho_{L} R_{S}\right]+\frac{\partial}{\partial z}\left[\rho_{L} R_{S} U_{L}\right]=-\Gamma,
\end{gathered}
$$

where $U_{G}=J_{G} / \alpha_{G}$ and $U_{L}=J_{L} / R_{S}$ are the velocities of the gas and liquid phases weighted by the volumetric fraction of each phase, with $\alpha$ the void fraction and $R_{S}$ the liquid fraction, $Q_{G}$ and $Q_{L}$ are respectively the volumetric flow rates of the gas and liquid phases. The variable $\Gamma$ is the interfacial mass transfer condition.

The equation of momentum conservation of the mixture, Eq. (6), is defined by [10]:

$$
\begin{array}{r}
\frac{\partial}{\partial t}\left[\rho_{G} \alpha_{G} U_{G}+\rho_{L} R_{S} U_{L}\right]+\frac{\partial}{\partial z}\left[C_{V, G} \rho_{G} \alpha_{G}\left(U_{G}\right)^{2}+C_{V, L} \rho_{L} R_{S}\left(U_{L}\right)^{2}\right] \\
=-\frac{\partial}{\partial z} P-\frac{f}{2 D}\left[\rho_{G} \alpha_{G}+\rho_{L} R_{S}\right] J^{2}-\left[\rho_{G} \alpha_{G}+\rho_{L} R_{S}\right] g \cdot \sin (\theta)
\end{array}
$$

where $C_{V, G}$ and $C_{V, L}$ are respectively the coefficients of covariance of the velocities of the gas and liquid phase, $\rho_{G}$ is the density of the gas, $f$ is the friction factor, $D$ is the inner pipe diameter, $P$ is the mixture pressure, $\theta$ is the column slop $\left(90^{\circ}\right)$ and $z$ is the axial coordinate along the pipe, varying from 0 to $L$. For turbulent flows the velocity and concentration profiles are approximately flat, such as the coefficient of covariance of the phase velocity is approximately equal to unity, $C_{V, k} \cong 1$. Thus, this coefficient is omitted from the momentum equation conversions in the formulations of the models defined in the sequence [9].

Considering the boundary conditions, the mass conservation equations of the gas and liquid phases, in the absence of mass transfer, $\Gamma=0$, are reduced to:

$$
\frac{d}{d z}\left[\rho_{G} \alpha_{G} U_{G}\right]=0 \quad \therefore \quad \rho_{G} \alpha_{G} U_{G}=G_{G}=\text { cte. }
$$




$$
\frac{d}{d z}\left[\rho_{L} R_{S} U_{L}\right]=0 \quad \therefore \quad \rho_{L} R_{S} U_{L}=G_{L}=\text { cte. }
$$

with the mass flow $G_{G}$ and $G_{L}$ of each phase remaining constant along the tube.

The simplification of Eq. (6), momentum conservation of the mixture for steady-state flow and $\sin \left(90^{\circ}\right)=1$ is given by:

$$
\begin{array}{r}
\frac{d}{d z}\left[\sum_{k=G, L} C_{V, k} \rho_{k} \alpha_{k}\left(U_{k}\right)^{2}\right]=-\frac{d}{d z} P-\frac{f}{2 D}\left[\rho_{G} \alpha_{G}+\rho_{L} R_{S}\right] J^{2} \\
-\left[\rho_{G} \alpha_{G}+\rho_{L} R_{S}\right] g
\end{array}
$$

Gathering the terms with the gradient in the axial direction, the Eq. (10):

$$
\begin{array}{r}
\frac{d}{d z}\left[P+\sum_{k=G, L} C_{V, k} \rho_{k} \alpha_{k}\left(U_{k}\right)^{2}\right]=-\frac{f}{2 D}\left[\rho_{G} \alpha_{G}+\rho_{L} R_{S}\right] J^{2} \\
-\left[\rho_{G} \alpha_{G}+\rho_{L} R_{S}\right] g
\end{array}
$$

The Eq. (10) can be written in terms of phase velocities, $U_{G}$ and $U_{L}$, with $\alpha_{G}+$ $R_{S}=1$ and $\rho_{s}=\rho_{G}+\rho_{L}$, so in a more simplified way:

$$
\frac{d}{d z}\left[P+\alpha \rho_{G}\left(U_{G}\right)^{2}+(1-\alpha) \rho_{L}\left(U_{L}\right)^{2}\right]=-\tau_{W, S}-\rho_{s} g
$$

where $\tau_{W, S}$ is frictional wall force the mixture per volume unit of the mixture. In the Eq. (11) shows the formulation of the mixture model, where there are in this Eq. 3 variables to be determined: $P, \alpha$ and $\tau_{W, S}$. And these variables are dependent on the flow pattern, in this case, bubble flow.

\subsection{Void Fraction}

The void fraction according to the flow pattern, in the case bubble flow, was determined from the volumetric balance as a function of the gas superficial velocity and the velocity of the dispersed bubbles. This void fraction model $\alpha$ is obtained from the slip kinematic law proposed by [11], Eq. (12):

$$
\alpha=J_{G} / U_{B}
$$

where $U_{B}$ is the bubbles velocity. According to the proposal of [13], which assumes that the bubbles move with the superficial velocity of the mixture $J$, plus the local slip speed $V_{\infty, B}[9]$.

$$
U_{B}=C_{0, B} J+V_{\infty, B}
$$

So that $V_{\infty, B}=C_{\infty, B}\left(\Delta \rho D g / \rho_{L}\right)^{1 / 2}$.

For dispersed bubbles, the distribution parameter $C_{0, B}$ and the dimensionless local slip velocity $C_{\infty, B}$ are defined in accordance with Eqs. (14) and 
(15) respectively. Considering the dispersed bubble regime, which results from the effects of surface tension, viscosity, inertia, and thrust [14].

$$
\begin{gathered}
C_{0, B}=1.0+0.2\left(1-\sqrt{\frac{\rho_{G}}{\rho_{L}}}\right) \\
C_{\infty, B}=\sqrt{2}\left(R_{S}\right)^{n} E^{-1 / 4}
\end{gathered}
$$

where $n=0$ for the agitated bubble regime and $n=7 / 4$ for a distocided bubble regime and considering the saturation condition $R_{S}=1-\alpha$. The variable $E o$ is the Eötvös number defined by $E o=\Delta \rho D^{2} g / \sigma$, with $\Delta \rho=\rho_{L}-\rho_{G}$.

Therefore, Eq. (12) can be written as a function of the volumetric fraction of liquid, such that:

$$
f\left(R_{S}\right)=1.0-R_{S}-\frac{J_{G}}{\left(C_{0, B} J+V_{\infty, B}\right)}=0
$$

where $f\left(R_{S}\right)$ is an implicit function of $R_{S}$, once $V_{\infty, B}$ is dependent of $C_{\infty, B}$, but $C_{\infty, B}$ is dependent of $R_{S}$ as shown on Eq. (15).

Therefore, the Eq. (16) is solved iteratively using a solution method of nonlinear equations, such as the secant method, at each step of integration $\Delta z$ from mixture model, in order to determine $R_{S}$ and other related variables.

\subsection{Friction Force Model}

The homogeneous model was used to estimate the value of $\tau_{W, S}$, since the flow pattern is limited to the bubble flow, because in this model it is considered a homogeneous mixture, applying the definition of a mixture viscosity and realizing the direct application of the single-phase friction factors.

Whereas $\tau_{W, S}$ the wall friction force per unit volume, it can be defined by the shear stress of the mixture $\tau_{W}$ multiplied by the ratio of the perimeter $S$ and the cross section area of the pipe $A$.

$$
\tau_{W, S}=\frac{\tau_{w} S}{A}=\frac{2 C_{f, s} \rho_{s} J^{2}}{D}
$$

where $C_{f, s}$ is the friction factor of the mixture Eq. (18) as a function of the Reynolds number of the mixture, $R e_{s}$, as defined in Eq. (19). These two variables are mixture dependent: the specific mass $\rho_{s}$ and viscosity $\mu_{s}$ of the mixture are, respectively, defined by Eqs. (20) and (21) according to [15].

$$
C_{f, s}=\left\{-3.6 \log \left[\left(\frac{\varepsilon}{3.7 D}\right)^{1,11}+\frac{6.9}{R e_{s}}\right]\right\}^{-2}
$$

where $\varepsilon$ is the absolute roughness of the pipe.

$$
\begin{gathered}
\operatorname{Re}_{s}=\frac{\rho_{s} J D}{\mu_{s}} \\
\rho_{s}=\alpha \rho_{G}+(1-\alpha) \rho_{L} \\
\mu_{s}=\alpha \mu_{G}+(1-\alpha) \mu_{L}
\end{gathered}
$$




\subsection{Mixture Model Solution}

The mixture model solution for a isothermal and steady state two-phase flow in bubble flow pattern is shown. By solving Eq. (10) the model is automatically solved. However, Eq. (10) was simplified to a reduced way of an ordinary, nonhomogeneous differential equation:

$$
\frac{d}{d z}[\Psi(z)]=-\tau_{W, S}(z)-\rho(z) \cdot g \cdot \sin (\theta)
$$

where $\Psi(z)=P(z)+G_{G} U_{G}(z)+G_{L} U_{L}(z)$ is an implicit function of $P(z)$. The variables of Eq. (22) are also dependent on $P(z)$ as shown in Eqs. (23), (24) and (25):

$$
\begin{gathered}
\rho(z)=\alpha(z) \rho_{G}(L)[P(z) / P(L)]+\rho_{L}[1-\alpha(z)] \\
U_{G}(z)=J_{G}(L)[P(L) / P(z)] / \alpha(z) \\
U_{L}(z)=J_{L} /[1-\alpha(z)]
\end{gathered}
$$

where $L$ is the position in the outlet section of the column where the variables are known. Therefore, the solution of Eq. (22) is conditioned in the solution of the equations that define $\alpha(z)$ and $\tau_{W, S}(z)$ which are also dependent on $P(z)$.

The solution of Eq. (22) is numerical integration using a Runge-Kutta fourthorder (RK-4) routine, programmed on MatLab. The integration step $\Delta z$ used was $D / 4$, where it was shown sufficient for the solution of the model. The variable $P(z)$ is determined by the iterative solution of the implicit equation $f(P)$, defined by:

$$
f(P)=P+G_{G}\left(U_{G}\right)_{\alpha}+G_{L}\left(U_{L}\right)_{\alpha}-\Psi=0
$$

The solution of the Eq. (26) can be obtained through the secant method, at each step of integration $\Delta z$. In others words, to solve the Eq. (22) it's necessary to solve at same time the Eq. (26), which means that for each sub-step of the $R K-4$, the Eq. (26) must be solved. On Matlab both methods were implemented/codified for a simultaneous solution, not resorting to any Matlab library function.

The mixture model solution is shown in Fig. 2. The input data is assigned at the beginning of the model, with the fluid properties and the geometrical conditions of the pipe. The flow pattern is determined. If the flow is a bubble flow, then the void fraction and friction force are calculated according to Eqs. (16) and (17). Other pattern flow beyond bubble flow will not be addressed in this article. The final computing is the pressure gradient, given by Eqs. (22) and (26).

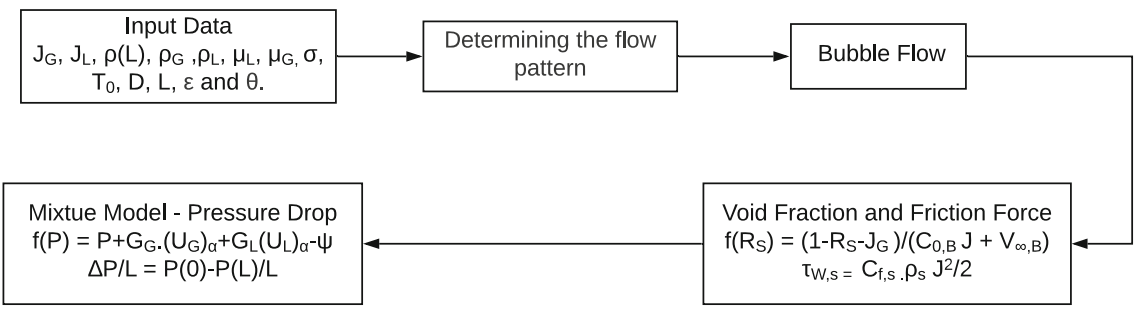

Fig. 2. Mixture model solution flow chart. 
The boundary conditions for the solution of the equations are the superficial velocities of the phases $J_{L}, J_{G}$ and the mixture pressure $P$. It is also necessary to define the flow temperature $T_{0}$ and the properties of fluids as the specific masses $\rho_{G}$ and $\rho_{L}$, the viscosities $\mu_{G}$ and $\mu_{L}$ and the interfacial tension $\sigma$. For the geometry of the pipe it is necessary to define the inner diameter $D$, the length $L$ and the absolute roughness, $\varepsilon$.

\section{Results and Discussion}

The experimental and theoretical study to validate the mixture model with 30 experimental data was performed for different gas and liquid superficial velocities: 6 superficial velocities of liquid $J_{L}$ varying from $1.720 \times 10^{-2}$ to $10.360 \times 10^{-2} \mathrm{~m} / \mathrm{s} ; 5$ superficial velocities of gas $J_{G}$ varying from $1.234 \times 10^{-2}$ to $2.072 \times 10^{-2} \mathrm{~m} / \mathrm{s}$.

\subsection{Void Fraction}

The void fraction analyses were made comparing the two experimental methods described in Sect. 2.2 for the quick-closing valves method and Zuber/Nicklin relation against the numerical results from the mixture model presented in Sect. 3.2.

The experimental void fraction data show for both methods, Figs. 3 and 4, respectively, for quick-closing valves method and Zuber/Nicklin relation that when the liquid flow is constant then the void fraction increases with the increase in gas input. For the opposite setup, fixed gas input and increasing liquid flow, the void fraction decreases as the superficial velocity $J$ of the mixture increases. This is a simple consequence of the higher fluid velocity in the pipe: the faster it moves, the faster it will carry the bubbles upwards. Such two-flow peculiarity was mentioned by [16] and was also observed in all experimental and numerical results. The model accuracy analysis is based on the relative deviation $\left(\delta_{\Phi}\right)$, Eq. (27), for the 30 experimental points performed.

$$
\delta_{\Phi}=100\left[\frac{\Phi_{n u m}-\Phi_{e x p}}{\Phi_{\exp }}\right]
$$

where $\Phi$ is a auxiliary variable represented as $(\Delta P / L)$ or $(\alpha)$.

These results are presented in Fig. 5 for the quick-closing valves method and Fig. 6 for Zuber/Nicklin relation. For the numerical results against the closing valves method, Fig. 5 , the mean error was $3.32 \%$; the mean minimal error for the lowest gas velocity of $1.451 \times 10^{-2} \mathrm{~m} / \mathrm{s}$ was $0.46 \%$ and mean maximal error was $9.52 \%$ for the highest gas velocity of $2.072 \times 10^{-2} \mathrm{~m} / \mathrm{s}$. The standard deviation of the relative error was $7.53 \%$ and the method shows a large variation of error according to the increase in gas velocity. For lower gas velocities the numerical method underestimates the results in more than $5 \%$ while for larger values of gas velocity it overestimates in excess of $13 \%$. This problem may be from the 


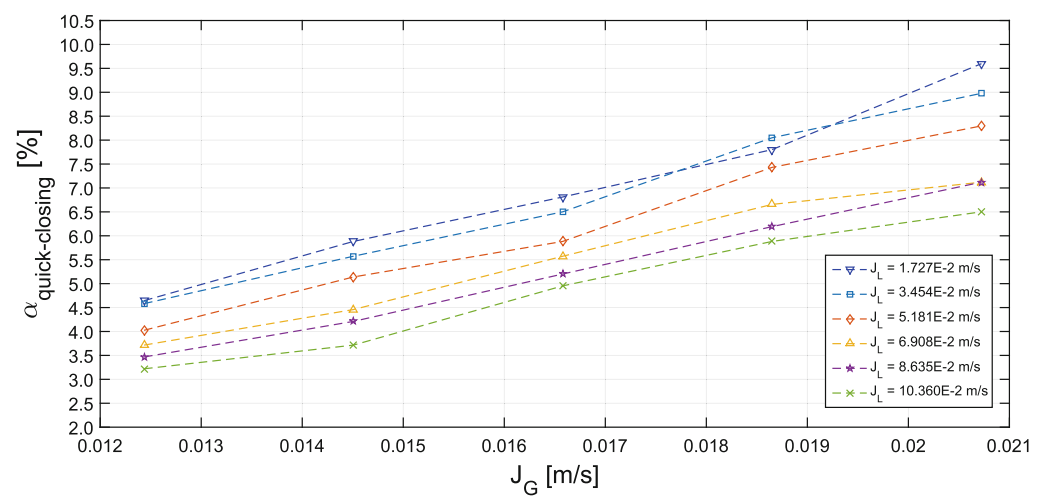

Fig. 3. Experimental results of void fraction for all $J_{L}$ tested for quick closing valves method.

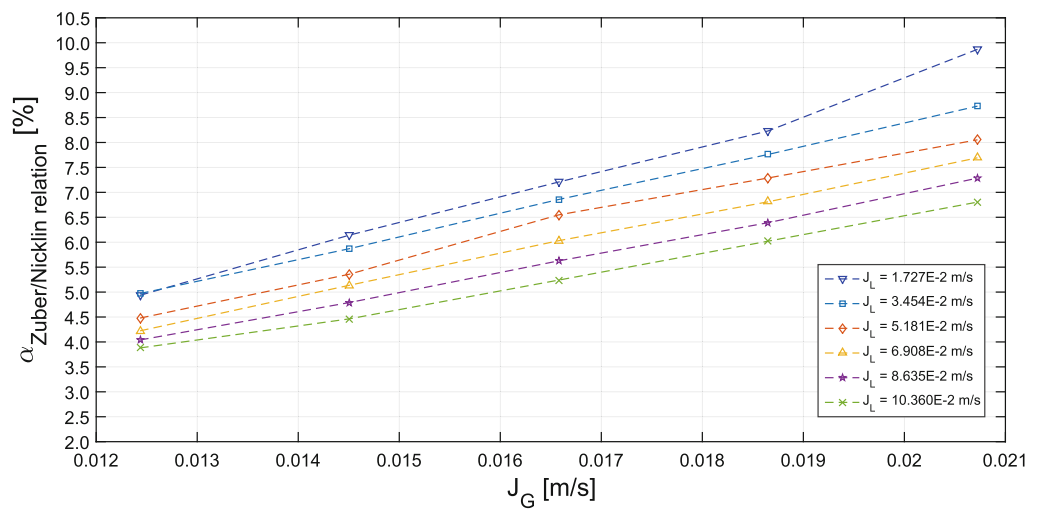

Fig. 4. Experimental results of void fraction for all $J_{L}$ tested for Zuber/Nicklin relation.

difficulty in ensuring the simultaneous manual closing of valves located more than $1.165 \mathrm{~m}$ apart. The numerical results against the Zuber/Nicklin relation method, Fig. 6, presented a mean error of 9.54\%. The error standard deviation was less than half of the quick-closing valves method, $3.67 \%$ against $7.53 \%$. Apart from one reading, all the numerical results overestimate the void fraction and the dispersion of the prediction is lower.

The bubble distribution parameter, required by this method, was considered $C_{0, B}=1$ for the experimental values, while it was individually calculated for every numerical type of flow, presenting an average value of $C_{0, B, \text { num }}=1.2$. The experimental value of $C_{0, B}=1$ is recommended by [10], and in fact, by calculating the void fraction for the experimental values using the mean numerical values of $C_{0, B, \text { num }}=1.2$, yielded much larger error values. 


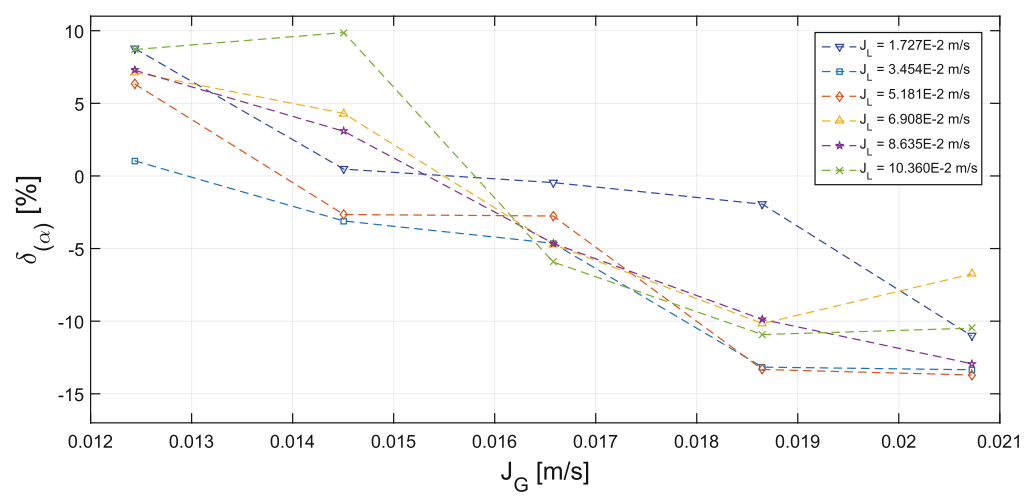

Fig. 5. Results for relative errors for all $J_{L}$ tested for quick-closing valves method.

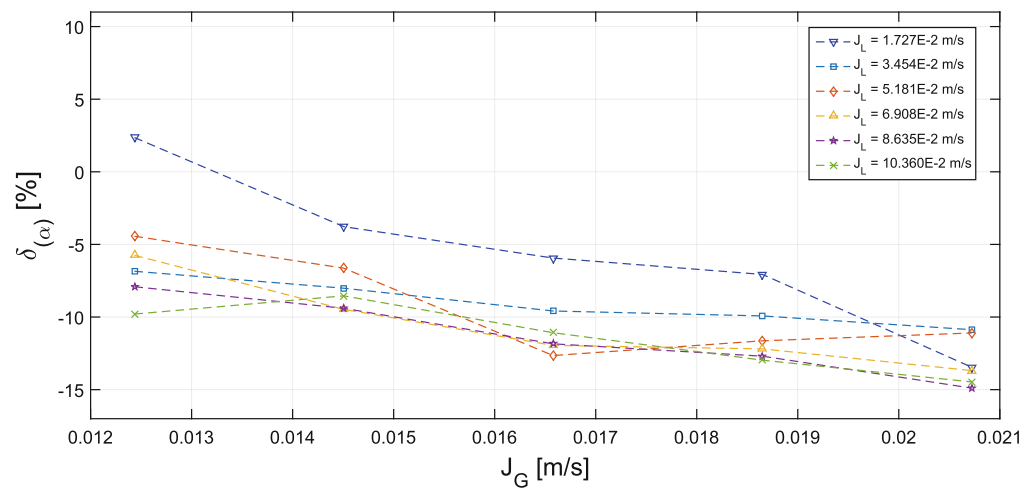

Fig. 6. Results for relative errors for all $J_{L}$ tested for Zuber/Nicklin relation.

\subsection{Pressure Gradient}

The pressure gradient analysis was made comparing the numerical results against the experimental results with a propagated uncertainty.

The pressure gradient for the lowest and highest fixed superficial liquid velocity and increasing superficial gas velocity are shown in Fig. 7. These curves presents a negative slope, that is, increasing the $J_{G}$, the void fraction also increases and the mixture density decreases also with the pressure gradient. Such feature was observed in all experimental and numerical results. The compared error of the experimental against numerical pressure gradient results, and respective uncertainties of the experimental pressure gradient measurements, are depicted in Fig. 8 for all superficial liquid velocities. The standard deviation of the relative error of the pressure gradient was $9.20 \%$, showing a stable correspondence between experimental and numerical results. 


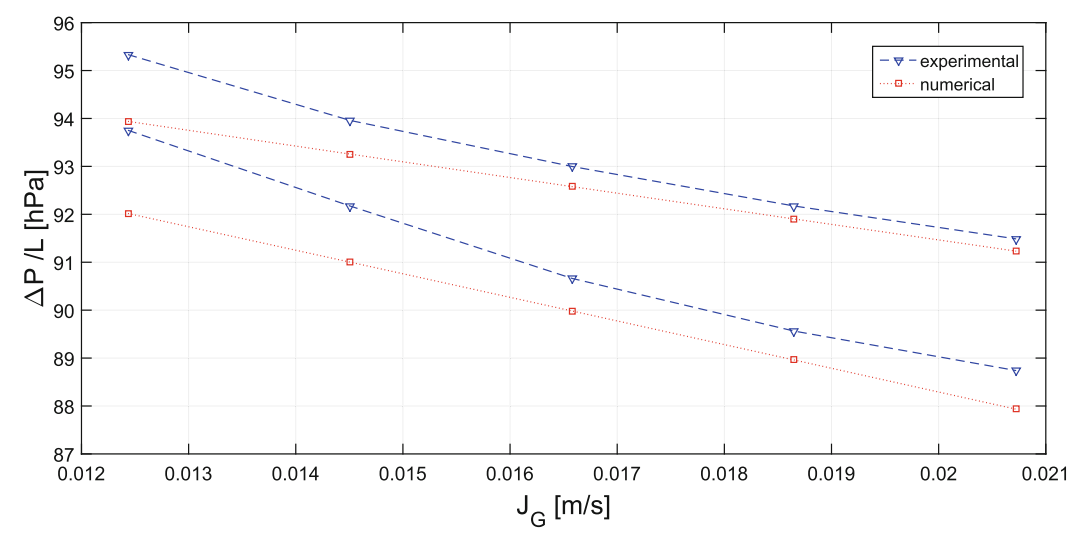

Fig. 7. Pressure gradient Experimental vs Numerical.

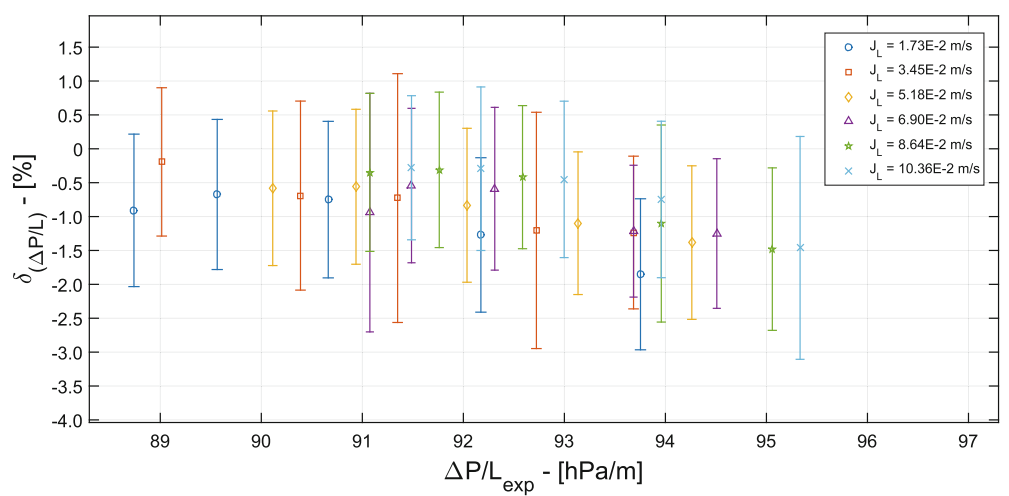

Fig. 8. Relative error from pressure gradient

As shown in Fig. 9, the relative error $\left(\delta_{(\Delta P / L)}\right)$ for the pressure gradients does not exceed $2.00 \%$ and the largest experimental uncertainty was $1.83 \%$. These low values were expected once this flow pattern is strongly influenced by the gravitational force, and dependent by the mixture density and void fraction.

The results were expected since the mixture model tends to make a good prediction of the pressure difference for this type of vertical bubble flow pattern as demonstrated by [9]. 


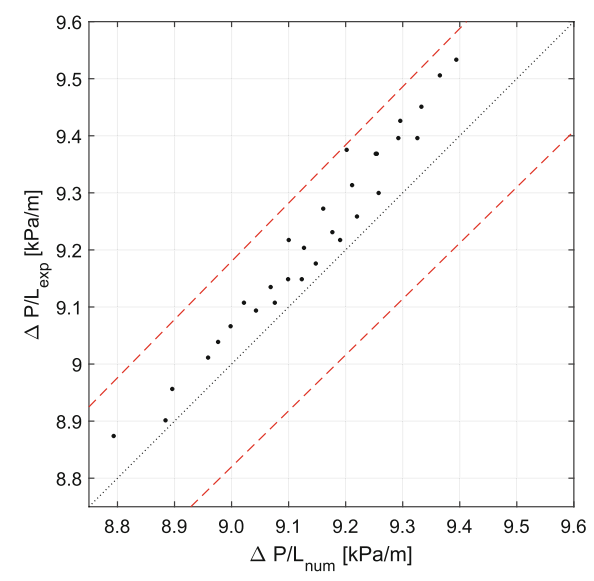

Fig. 9. Pressure gradient $\Delta P / L$ comparison for experimental results (y-axis) against numerical results (x-axis), dashed red lines correspond to the upper and lower $2 \%$ experimental error.

\subsection{Other Challenges and Improvement Opportunities in the Experimental Apparatus}

The quick-closing valves method was effective and simple, but it's accuracy could be improved with solenoid valves to guarantee the simultaneous closing by only one operator. Also the experimental void fraction determination via the Zuber/Nicklin relation method could also be improved by changing the velocity measurement procedure: from a manned chronometer to measure the bubble ascending time to an electronic/optic velocity sensor.

The experimental gradient pressure measurement was simple and reliable when the connection from the hoses between the bubble column and the differential manometer was non-obstructed. Other wise, $\Delta P / L$ was unreliable.

It was found that the gas rotameter, R3 in Fig. 1(a), is more accurate when operating in medium range, approximately half of its scale. An observable consequence is that the relative percentage error of $\Delta P / L$ was the lowest when the gas rotameter was in the middle scale, independently of the liquid flow, Sect. 4.2.

Other types of flow patterns may be obtained in this apparatus. The provided examples of slug flow Fig. 10(a), churn Fig. 10(b) or annular Fig. 10(c) were photographed in the IPB's two-flow experimental apparatus. Furthermore, it is also possible to be prepare the apparatus for downward, counter-current, co-current and U-tube flow research. 


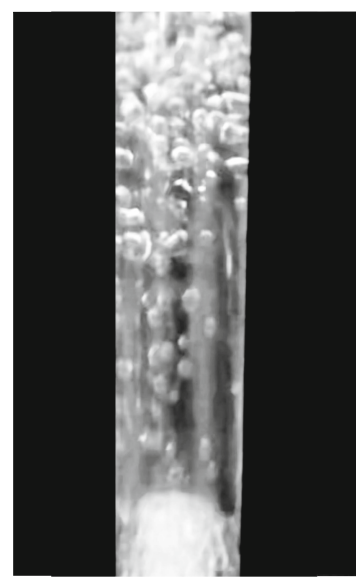

(a)

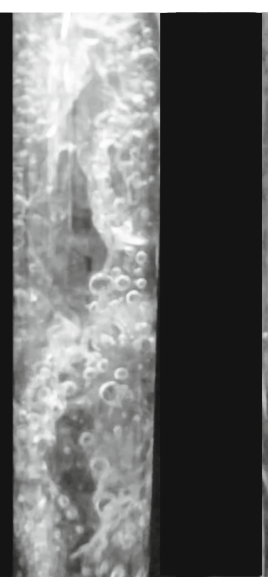

(b)

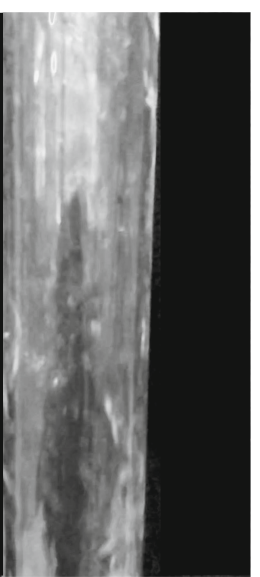

(c)

Fig. 10. Other two-phase flows captured from IPB's apparatus: (a) Slug flow; (b) Churn flow; (c) Annular flow.

\section{Conclusions}

A vertical, upward air-water experimental and numerical study of bubble flow pattern is presented via 2 relevant parameters: the pressure gradient and the void fraction. The two-phase flow conditions were: one dimensional, steady-state, isothermal, no phase transition, no mass transfer and constant specific mass and viscosity.

The mixture model was the numerical solution implemented to obtain the theoretical pressure gradient and void fraction. Its solution is based on analytical equation to predict closing parameters.

The experimental results of the pressure gradient were obtained with a differential manometer and the void fraction was obtained with two methods: quickclosing valves method and Zuber/Nicklin relation. The comparison of the experimental against numerical parameters was performed for 30 pair of superficial velocities. The relative error for pressure gradient envelope was inferior to $2.00 \%$ and the largest experimental uncertainty measured was $1.83 \%$. The results of void fraction with quick-closing valves method had a medium relative error of $3.32 \%$ and for Zuber/Nicklin relation was $9.54 \%$.

The newly rebuild laboratory apparatus is reliable, although it presents conditioning set-up particularities regarding operational procedures and fine-tuning, that have to be accounted for. 


\section{References}

1. Huang, F., Takahashi, M., Guo, L.: Pressure wave propagation in air-awater bubbly and slug flow. Prog. Nucl. Energy 47, 648-655 (2005)

2. Hanafizadeh, P., Ghanbarzadeh, S., Saidi, M.H.: Visual technique for detection of gas-liquid two-phase flow regime in the airlift pump. J. Pet. Sci. Eng. 75, 327-335 (2011). Elsevier BV

3. Kassab, S.Z., Kandil, H.A., Warda, H.A., Ahmed, W.H.: Air-lift pumps characteristics under two-phase flow conditions. Int. J. Heat Fluid Flow 30, 88-98 (2009)

4. Schlegel, J., et al.: Void fraction and flow regime in adiabatic upward two-phase flow in large diameter vertical pipes. Nucl. Eng. Des. 239(12), 2864-2874 (2009). Elsevier BV

5. Chalgeri, V.S., Jeong, J.H.: Flow patterns of vertically upward and downward airwater two-phase flow in a narrow rectangular channel. Int. J. Heat Mass Transf. 128, 934-953 (2019). Elsevier BV

6. Szalinski, L., et al.: Comparative study of gas-oil and gas-water two-phase flow in a vertical pipe. Chem. Eng. Sci. 65(12), 3836-3848 (2010). Elsevier BV

7. Xue, Y., et al.: Investigation on the void fraction of gas-liquid two-phase flows in vertically-downward pipes. Int. Commun. Heat Mass Transf. 77, 1-8 (2016). Elsevier BV

8. Taitel, Y., Bornea, D., Dukler, A.E.: Modelling flow pattern transitions for steady upward gas-liquid flow in vertical tubes. AIChE J. 26, 345-354 (1980)

9. Lima, L.E.M.: Análise do Modelo de Mistura Aplicado em Escoamentos Isotérmicos Gás-Líquido, Ph.D. thesis, Universidade Estadual de Campinas (2011)

10. Pauchon, C., et al.: Tacite: a transient tool for multiphase pipeline and well simulation. In: Society of Petroleum Engineers (1994)

11. Zuber, N., Findlay, J.A.: Average volumetric concentration in two-phase flow systems. J. Heat Transf. 87(4), 453-468 (1965)

12. Wu, B., Firouzi, M., Mitchell, T., Rufford, T.E., Leonardi, C., Towler, B.: A critical review of flow maps for gas-liquid flows in vertical pipes and annuli. Chem Eng J. 326, 350-377 (2017). https://doi.org/10.1016/j.cej.2017.05.135

13. Nicklin, D.J.: Two-phase bubble flow. Chem. Eng. Sci. 17(9), 693-702 (1962). ISSN 0009-2509

14. Ishii, M.: Thermo-Fluid Dynamic Theory of Two-Phase Flow. Eyrolles, Paris, France: [s.n.]. Collection de la Direction des Etudes et Recherches d'Electricite de France (1975)

15. Dukler, A.E., Wicks, M., Cleveland, R.G.: Frictional pressure drop in 2-phase flow: B. An approach through similarity analysis. AIChE J. 10(1), 44-51 (1964). ISSN 0001-1541

16. Wallis, G.B.: One-Dimensional Two-Phase Flow. McGraw-Hill Book, New York (1969). [S.l.] 